Environment Conservation Journal 16 (SE) 181-184 , 2015

ISSN 0972-3099 (Print) 2278-5124 (Online)

Abstracted and Indexed

\title{
Visual and Ecological Prerequisites of Development of Modern Architecture
}

\section{Dubinskii Vladimir Petrovich}

\author{
Received:25.08.2015
}

Revised:30.08.2015

Accepted:30.09.2015

\begin{abstract}
Visual and ecological prerequisites for the development and formation of modern architecture, taking into account the impact of global crises have been stated in the present research.Objectives: to identify the prerequisites and principles of visual-ecological formation of modern architecture taking into account the influencing factors.
\end{abstract}

Keywords:modern architecture, visual ecology, form making, global crises.

\section{Introduction}

Today we can talk about the end of yet another stage of modern history that shows us itself in all its variety and diversity, ambiguity and contrast. Global disasters, adverse environmental predictions, unstable political and economic situation are found side by side with the impressive scientific and technological progress, "information explosion" and improvement of the quality of life. In this regard, there is an increase in the instability of global development. The situation of gaining of uncertainty, entropy and chaos in the world is naturally reflected in the modern culture: philosophy, art, architecture. [Holodova,2004].

However, as it always happens in history, this instability leads to the emergence of new interesting phenomena, in architecture in particular. Many scientists studying different fields of knowledge have repeatedly noted that the processes taking place in society, science, the arts, architecture are largely governed by the laws of development formulated by biologists. The laws of evolution, energy conservation, the law of minimum and so on, if to study them carefully, manifest their properties in many areas of human activity. It is also very important to understand the mutation of systems under the influence of numerous crises. It becomes especially visible due to the fact that the crisis frees a lot of space i.e. ecological niches; the stabilizing selection weakens sharply; the destabilization of adaptive norms happens followed

\section{Author's Address}

Cand.Sc.Arch. Professor of the Faculty of architecture of buildings and con-structions of Kharkov Beketov National University of Municipal Economy by the increase of variability and the rapid form form making. The evolution in these conditions becomes very fast and barely predictable. Though, of course, evolution cannot become completely unpredictable and "random". It is because all possible ways of evolutionary changes are predefined (channeled) toughly by the structure of the organism and its ontogenesis. Only one of the two main limiting factors of evolution and guides is being removed - the ecosystem factor; the second one - the organismic, or "epigenetic" - remains.

From this perspective, the revolutionary changes of architectural forms of the last two decades have become possible due to the opening of new niche of development. This is a process of deep qualitative change in the composition of these forms, their relationships and structures of functioning. The emergence of this niche has been determined by several powerful factors including: demographic crisis, technological and information revolution, the emergence of new paradigms and innovations in the architecture.

\section{Results}

The development occurs in two forms - the evolutionary and revolutionary (hopping) one, both of them can be viewed as phases of the development. In the evolutionary phase the process of changes in time of the architectural forms at various levels, relations between them and their functioning usually varies slightly and smoothly. Gradually the oscillations of the formal parameters of the architecture are being enhanced. When the value of fluctuating parameters exceeds the critical 
value and the power of stabilizing systems, there comes a moment when an arbitrarily small change in the parameters leads to an abrupt transition of the architectural language into a qualitatively different state. This is how the point of bifurcation emerges, bringing with it the moment of branching options for the development of architecture, the revolutionary phase. Near the bifurcation point the architecture is more responsive to all impacts i.e. even the smallest fluctuations may cause major changes; that is why the adaptation period is one of the most important parts in its development.

One of the main features of the biosphere lies in the fact that the price of stability and variability, plasticity and development of all that exists in the biosphere is the death of any organism of those who are living now and will live subsequently. We witness this incessant death and rebirth in the flow of history, when the whole ethnic groups are being killed and instead new ones launch, when civilizations are dying and are being replaced by other, when states die and news states arrive on the place of the previous ones, and new, unusual and not always understandable and familiar architectural styles and directions are emerging in time.After each crisis, the new system is being composed of fragments of the old, destructed biosphere. And this new biosphere usually appears to be more complex, diverse and stable than the former one. The disasters that had caused mass extinctions hundreds of millions of years ago may not have had such an effect in modern world. In addition, the speed of progressive evolution in the peaceful era between crises is gradually increasing. This process is embodied in innovative architectural styles. For example, speaking about modern urbanism, we can say that the city becomes a system or an organism that expresses the multiplicity of content and links, coexisting together in the same urban structure. Technical innovations, scientific achievements, demographic growth, economic development, the formation of new cultural models - all this together determines new forms and models of the environment. Modern city is a structure that recites itself, it doesn't stay fixed, but changes and mutates.

The eagerness of the architecture to make a breakthrough to a new logic, assigned by a new anti-dogmatic, open-minded, "risking" science (Bachelard), is reflected not only in the "risking" concepts of the leading theorists of architecture, but also in the reflections of architects, often taking the form of a manifesto. For instance, Cecil Belmond writes: "We are trying to break free from the shackles of conventional structures. Our buildings have to be constructive. But they have to get read of the subordination by a Cartesian logic, in the name of which we are dissecting the space with strict horizontals and verticals ..., closeness in right angles assumes understanding the order as a strict linearity. The closeness contains the idea that the building-construction first of all is the object... the form is understood as completed and strictly regulated concept of the order. This is accepted as the status quo. The imagination here stands still. And we ourselves have set these traps on the roads that are leading us to the motion". [Rybaltovskaya] The striving after a renovation forces the architect to appeal to a new science, to the philosophical reflection over the essence of the changes occurring in the thinking process. Developing the concept of the "new structure" and "non-rigid form (formlessness)", Belmond writes: "To my great happiness, a new science had offered us new endeavors. By rejecting the linear "pre-owned" logic of hierarchy of "top-to-the-bottom" in the process of reasoning, it had opened the tantalizing prospect of complexity. The nonlinearity has already taken a strong position. The access to the feedback is new and apparently, the main idea of the process. The access is defined as a main motive, main driving force behind all natural processes ... Achievements of a new science are based on the notion of living dynamical systems. "[Rybaltovskaya]

The challenge lies in the fact that the notion of dynamism cannot be moved directly into the architecture, because architecture cannot exist outside a fixed form. Architect has to carefully scrutinize the new paradigmatic construction that is customized to the conscious choice of the way of risk-taking, to the internal dynamic process of formation, which itself will bring the process to the point of a conflict.However, conflicts of this nature can lead to conflict in the perception of a person who receives such a visual environment.

And here it is necessary to appeal to such a concept as homeostasis. In biology, the homeostatic mechanism keeps the system from both premature death and the excess growth. 
Together with the increasing complexity of the system happens the lowering of its stability, and life of the biosphere depends on the stability and variability, their optimal combination is called plasticity. One of the basic principles, reflecting the state of stability is the principle of homeostasis. Homeostasis is a pursuance of preserving the essence of the system, its immutability. If the system is too homeostasis, if it is almost unable to be changed, then the change in external conditions, very often after the futility of the mobilization of all internal potentials, it is destroyed either under the attack from the outside or from the internal overpressure. For self-preservation system needs to have a variability option. However, if the potential to the variability is too big, then this ability can undermine the system from within, which leads to self-destruction of the system. The definitive combination of stability and variability, called plasticity, ensures the sustainable development of the system.

"Ecological system" behaves rationally - it has a "purpose" - a balance with the environment. Ecological systems act similarly, either adapting to any environment, or being extinct. It should be borne in mind that the adjustment of the trajectories of the system development is being made through the adjustment of goals of this system. In natural systems clear hierarchy of objectives is defined, and there always is the main objective i.e. refinement to the biosphere cycles, refinement of the system into the super-system. Thus, natural systems perform one of the most important principles - the principle of co-development of the systems. By calculating many options using the accumulated structural information, the system is selecting those that meet the criteria of preservation of stability and consistency to the super-system goals.However, if the rest of the natural world lives under the law of submission to the environment, to its inner laws, human, on the contrary, subordinates the environment to himself, thus violating one of the most important principles - the principle allowing the system to discard those elements and structures, and objectives of which are contrary to its own. This is one of the most important properties of the system to be used in the present study. Techno sphere, and the architectural environment is its part, shall correlate its objectives with the biosphere as its super-system, enter its technology to the cycles of biosphere to preserve the basic characteristics of the natural and human environment. Thus, in case of creation of sustainable, non-violent symbiosis between the techno sphere and the biosphere, this process, in terms of "visual ecology of architectural environment", would be perceived by humans as an aesthetic constant.

In this aspect, the most important issue is the clarification of the mechanisms ща generating ща the systematic architectural environment of cooperation with its main consumer - human being. Drawing parallels between the architecture and living ecological systems, we note that any living system should have a resistance in order to survive. By the resistance we understand the ability of the system to maintain its own homeostasis. Living systems definitely possess this skill. Moreover, with the flow of the evolution the sustainability of the living systems is growing steadily. Stability is in primis the ability of the system to adapt to changing conditions by means of an appropriate modification of its own structure. The revolutionary restructurings, as we know, are fraught with trouble. Too often nobody wants to make revolutions, and rightly so.

However, it is not possible to dispense with revolutions at all, neither society nor nature have succeeded without it. In order a fundamentally new system to emerge, the old structure shall be destroyed, and then, something new can be built from its wreckage.The rule of "intermittent equilibrium" (the transition to a new norm through the destabilization of the old one) is evident not only in the evolution of species, but also in the development of entire communities as well as in the development of art and architecture. Innovative architectural sites today go beyond the scope of usual order of architectural project, taking the form of complex-shaped structures and symbolic transformations that represent a hierarchy of forms and meanings on a new level of perception in conjunction with traditional compositional techniques of the artistic language of architecture. These structures are questioning, destroying the standard idea of orderliness, normative unity and usual subordination of the image to the functional or typological constituent. Composite priorities enter qualitative contradiction with the functional 
requirements, as well as with the manifestation of their essence in the figurative features of buildings. According to I. Dobritsina, the innovative architectural phenomena of the last three decades in their internal relationships and close connection to the philosophical systems are holding those very implicit common grounds that keep and even strengthen the pluralistic situation in architecture. Contemporary avant-garde architecture is that very edge that protects itself from diving into the abyss of chaos, which is so usual in the consumer society, where the selection mechanism is taking into account only immediate benefits, stylization and image-making. Striving of architecture as a system to the state of sustainability requires continuous improvement of its own mechanisms of development. The scientific approach to this problem is able to open in the spontaneity of this development a certain kind of consistency and thus to predict and maybe adjust the aesthetic and visual style, and ultimately the quality and harmonic architectural environment [Dobritsina2004].

Similarly, the advanced community with its complex structure of food chains, with many interdependent ecological niches, each of which is taken by a certain specific kind - is the same stable system, as well as the organism, and it is not always capable of smooth, gradual changes.

\section{Conclusion}

Conclusions that can be drawn from the above facts are very interesting. Techno-futurism of 60 -ies of the 20th century, where the reclamation of Earthlike planets of the Universe has become the main theme of fantasies of a mankind, is senseless in terms of the biological and social expediency.

Human adaptation to the conditions that are different from the terrestrial will produce a new type of Homo sapiens physiologically quite different from the existing that in turn will create social patterns and relationships alien to humanliving on Earth. That is, for humanity as a whole, at this stage of development, the external expansion may be only of scientific interest, not an economic one. At the same time changes in the environmental conditions on the planet itself may create the conditions for changing similar to the experiment of entomologist G. H. Shaposhnikov when the change in the chemical composition of air, food and water would have determined the type of mutation of species. Naturally, this process may take centuries, but if not to take any action now, the consequences of technical activities of a mankind cannot be predicted. We allot to the architecture a special role in the stabilization of the crisis, as this kind of human activity provides the environment favorable for the functioning of the human kind.

\section{References}

Dobritsina I. A. To the methodology of research of innovations // «Academia. Building and architecture». - M.: RAASN, 2004, No 2. - P. 16-18.

Holodova L. P. Professional architectural education: two schools and two global styles / L. P. Holodova // continuing architectural education: challenges and perspectives: materials of international scientific conference. 19-25 $5^{\text {th }}$ September 2004., Volgograd / VolgSUACE. - Volgograd, 2004. - P. 133-135.

Rybaltovskaya E. Modern architecture rests on invisible creatures. Building and immovable property. http://www.nestor.minsk.by/sn/index.html. 